\title{
Active optics in deployable systems for future earth observation and science missions
}

Ludovic Canas, Stéphanie Behar-Lafenêtre, Christophe Devilliers, Laurent Perrona, Lauriane Galtier, et al.

Ludovic Canas, Stéphanie Behar-Lafenêtre, Christophe Devilliers, Laurent Perrona, Lauriane Galtier, David Boban, Nicolas Tetaz, Guillaume Butel, Aurélien Suau, Jérôme Demilly, Stéphane Vezain, Frédéric Falzon, JeanFrançois Sauvage, Laurent Mugnier, Noah Schwartz, Ruben SanchezJanssen, Marc Ferrari, Mamadou N'Diaye, Pascal Hallibert, "Active optics in deployable systems for future earth observation and science missions," Proc. SPIE 11852, International Conference on Space Optics - ICSO 2020, 118522H (11 June 2021); doi: 10.1117/12.2599380

SPIE Event: International Conference on Space Optics - ICSO 2021, 2021, Online Only 


\section{International Conference on Space Optics-ICSO 2020}

Virtual Conference

30 March-2 April 2021

Edited by Bruno Cugny, Zoran Sodnik, and Nikos Karafolas
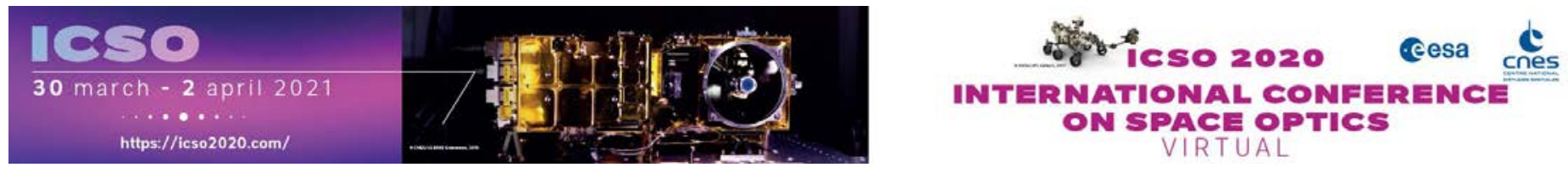

\section{Active optics in deployable systems for future earth observation and science missions}

\section{Cesa isopmeatians ecnes}




\title{
ACTIVE OPTICS IN DEPLOYABLE SYSTEMS FOR FUTURE EARTH OBSERVATION AND SCIENCE MISSONS
}

Ludovic Canas $^{1}$, Stéphanie Behar-Lafenêtre ${ }^{1}$, Christophe Devilliers ${ }^{1}$, Laurent Perrona ${ }^{1}$, Lauriane Galtier ${ }^{1}$, David Boban ${ }^{1}$, Nicolas Tetaz ${ }^{1}$, Guillaume Butel ${ }^{1}$, Aurélien Suau ${ }^{1}$, Jérôme Demilly ${ }^{1}$, Stéphane Vezain ${ }^{1}$, Frédéric Falzon ${ }^{1}$, Jean-François Sauvage ${ }^{2,4}$, Laurent Mugnier ${ }^{2}$, Noah Schwartz ${ }^{3}$, Ruben Sanchez-Janssen ${ }^{3}$, Marc Ferrari ${ }^{4}$, Mamadou N'Diaye ${ }^{5}$, Pascal Hallibert ${ }^{6}$

${ }^{1}$ Thales Alenia Space, 5 Allée des Gabians, 06150 Cannes, France

${ }^{2}$ Office National de Recherches Aérospatiales, 29 Avenue de la Division Leclerc, 92320 Châtillon

${ }^{3}$ UK Astronomy Technology Centre, Blackford Hill, Edinburgh EH9 3HJ, United Kingdom;

${ }^{4}$ Aix Marseille Univ, CNRS, CNES, LAM - Laboratoire d'Astrophysique de Marseille, 38 Rue Frédéric Joliot Curie, 13013 Marseille, France

${ }^{5}$ Université Côte d'Azur, Observatoire de la Côte d'Azur, CNRS, Laboratoire Lagrange, Bd de l'Observatoire, CS 34229, Nice Cedex 4, France;

${ }^{6}$ European Space Agency, Keplerlaan 1, NL-2200 AG Noordwijk

\begin{abstract}
This paper describes the outcomes of a study funded by the European Space Agency aimed at identifying the technical challenges and trade-offs at the system level, performing preliminary designs of an active correction loop for large deployable telescopes, and defining technological roadmaps for the development of the active correction loop for the selected designs.

This study has targeted two very different application cases, one for High Resolution Earth Observation from Geostationary orbit (called GeoHR, with a $4 \mathrm{~m}$ diameter entrance pupil) and one for Science missions requiring very large telescopes (with a up to $18 \mathrm{~m}$ diameter entrance pupil) with high-contrast imaging capabilities for exo-Earth observations and characterization.

For both application cases, this paper first summarizes the mission objectives and constraints that have influence on the telescope designs. It then presents the high-level trade-offs that have been led and the optical and mechanical design that have been developed, including the deployable aspects.

Finally, the performance assessment is presented, and is the basis for the justification of an active optics correction chain, with a preliminary set of requirements for typical components of the system. The presentation is concluded with proposed technological roadmaps that aim to allow the development of the building blocks on which the next generation instruments will be able to rely on.
\end{abstract}

Keywords: telescope, deployable mirror, active optics, deformable mirror, wave front sensor, positioning mechanism, segmented mirror

\section{INTRODUCTION}

Thales Alenia Space has been designing and developing space optical instruments for more than 40 years. Thales Alenia Space is now a leader in active optics for space, at all levels: system, building blocks, validation and tests. It has taken one step closer to future systems with deployable elements and active optics with a team of world-leading partners: ONERA (Office National d'Etudes et de Recherche Aérospatiales) is a world leader in High Angular Resolution imaging, in particular in the field of adaptive optics for ground application and active optics for space application. The role of ONERA in this project is to specify the Active optics system for Science case application.; UKATC is the UK's national laboratory for the design and development of astronomical instrumentation and is a world leader in managing 
and building space and ground based instrumentation and systems ; LAM is one of the three largest space laboratories in France, combining astrophysics with research and development in optics, including active/adaptive optics, for groundand space-based instruments ; the Observatoire de la Côte d'Azur (OCA) is an internationally renowned research center in Earth and Universe sciences, with broad expertise in astronomy and related instrumentation for ground-based and space telescopes.

\subsection{Scope and purpose}

Increasingly demanding space-based applications require a large primary mirror diameter, on which depends the optical instrument resolution. However, mass and volume of a monolithic primary mirror would dramatically increase along with its size, which, in addition to cost and technical issues, is also limited by the launch vehicle fairing available volume. Thus, to reach large primary mirror diameters, development of instrument that will include a deployable, segmented primary mirror and an active correction loop to optimize its optical performance will be of utmost interest. Such technologies will allow addressing highly demanding space-based applications such as high-contrast imaging for Earth-like exoplanets direct detection or high resolution Earth observation from the geostationary orbit.

This study aims at defining a technological roadmap of such an active optics correction loop, which will include sensing devices and active correction components. This technological roadmap will be supported by the design of two active correction loop which will be implemented in two preliminary instrument designs dedicated to two different missions:

- A Science mission case, the goal of which is the imaging and characterization of Earth-like exoplanets;

- An Earth observation mission at high-resolution case, from the geostationary orbit.

For both cases, the active optics design has been performed through trade-off analysis of correction strategies and technologies, which will be based on the requested performances evaluation at system level. Such an evaluation will allow identifying the active optics correction chain technical challenges and proposing a development roadmap.

\subsection{Earth Observation case}

The way to proceed has been to systematically analyze the potential combinations of active optics components, in order to identify the ones that can be studied. The systematic approach is beneficial to ensure that every interesting case is not forgotten and that any case which would not be intuitively considered interesting is also evaluated. The definition of an interesting case is discussed later in this paper, where we propose the criteria chosen to select or dismiss configurations.

\subsection{Science case}

Futur fundamental Science missions like LUVOIR (Large UV/Optica/Infrared Surveryor) shall be with a large international sharing funding and technological developments such as a complete Primary Mirror system. They shall be within a Science Objectives approach and disruptive from the JWST technologies already close to their asymptotic limit and too far from the LUVOIR-A Ultra Stability needs (10pm / 10mn stability).

\section{ACTIVE OPTICS IN DEPLOYABLE SYSTEMS FOR EARTH OBSERVATION}

\subsection{Summary of requirements}

The active optics correction loop will here be used to guarantee the MTF requirements in PAN and multispectral channels by:

- Performing the telescope WFE correction during the telescope commissioning, in particular the segmented primary mirror cophasing after deployment;

- Guaranteeing the telescope image quality for observation through LoS stabilization and WFE compensation, including notably the primary mirror segment cophasing.

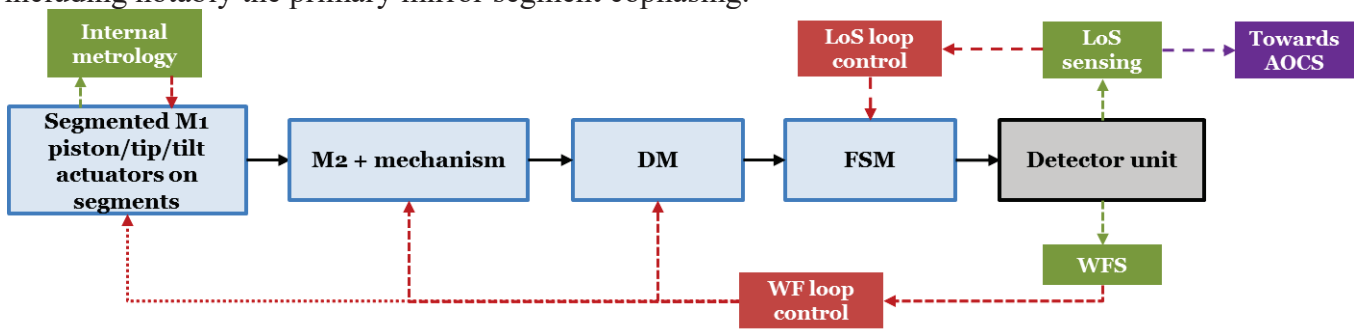

Figure 1: Functional diagram of the active optics loop strategy for the EO mission case. 
This active optics loop for the EO mission case relies on the following elements:

- Correction components: Actuated primary mirror segments to ensure the primary mirror cophasing, secondary mirror mechanism to relax the mechanical constraints on the secondary mirror positioning, DM (Deformable Mirror) to compensate for the telescope WFE (Wave Front Error) and FSM (Fast Steering Mirror) to ensure the LoS (Ligne-of-Sight) stabilization.

- Sensing: Internal metrology technologies may be used to maintain the primary mirror cophasing (after commissioning), while WFS (Wave Front Sensor) and LoS sensor will be required to assess both WFE and LoS errors.

- Control algorithms: LoS control will require a dedicated control algorithm that will compute the FSM position from the LoS sensor measurements, while another algorithm will be used to control both M2M (Segondary Mirror Mechanism) and DM to ensure the telescope WFE minimization. A last algorithm will be required to actuate the primary mirror segments from the wavefront sensor position, at least for commissioning operations.

\subsection{Trade-Off of System Level Architecture}

By a systematic approach, we set up a telescope only considering 3 main optical planes and 1 optional optical plane:

- The entrance pupil plane (equivalent to the primary mirror plane),

- The secondary mirror plane,

- The exit pupil plane,

- And, as an option in some designs, the intermediate pupil plane.

The defined parameters are the following:

- Presence of a mirror in the plane

- Present mirror is segmented

- Present mirror is active

- Active correction is at the micrometer level (MLA), at the nanometer level(NLA) or at both levels.

We end up with 576 possible cases with the 3 main planes and 5184 cases with 4 planes.

We used systematic criteria to reduce the cases to a few hundred and then case by case criteria to bring it down to 10 .

Below the criteria that have been retained, considered interesting for this study:

- Primary mirror has to be segmented,

- Primary mirror is not corrected only at nanometer level,

- Secondary mirror is always corrected and, at least, at micrometer level,

- Exit pupil always has a correction,

- Exit pupil, if monolithic, does not have only a micrometer correction (M2 has it already)

\subsection{Optical Design Trade-Offs}

To limit the optical computation we selected the following criteria:

- Simplicity of AIT

- Potential to reach the expected performance

- Capability to deploy M1

- Complexity to deploy M2

- Availability of operational time

- Actuators technology readiness level

- M1, M2 technology readiness level

- Complexity of the loop

- Potential for redundancy

- Potential for compensation of focal plane wrong positioning

Finally 2 solutions were identified as promising: 
- Case ${ }^{\circ}$ 3: This configuration has a M1 mirror constituted by six active segments. Each segment is moved by hexapod (MLA). M2 mirror is monolithic and is also moved by hexapod (MLA). There is one deformable mirror in the exit pupil. This mirror is formed of 6 segments. Each segment is the conjugated of a M1's segment. Each segment is also displaced by hexapod.

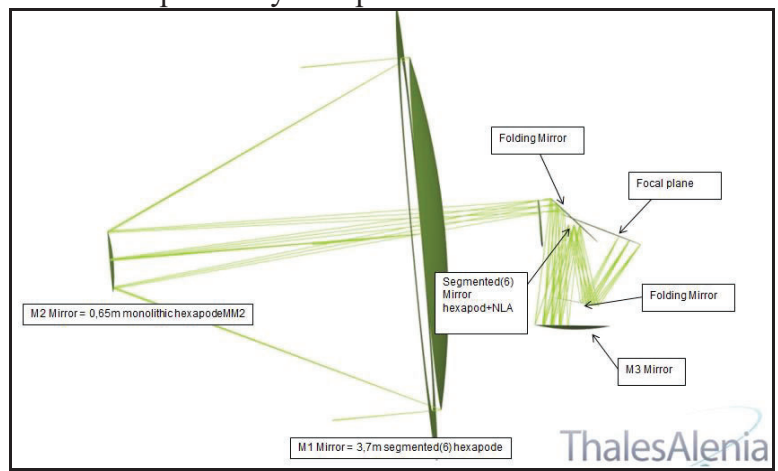

Figure 2: Optical combination $\mathbf{n}^{\circ} 3$

- Case $\mathrm{n}^{\circ}$ 8: This configuration has a M1 mirror constituted by six segments with no mechanism. The deformable segmented mirror, in the exit pupil, is not mounted on hexapods. Thus there is a segmented plane mirror mounted on hexapods in the intermediate pupil.

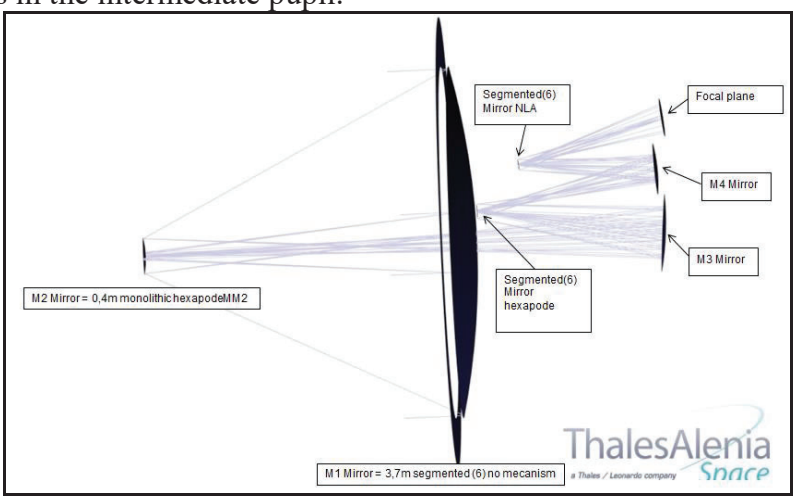

Figure 3: Optical combination $\mathbf{n}^{\circ} 8$

\subsection{Performance Assesment}

We have simulated in $\mathrm{CodeV}$ the wavefront at the exit pupil level, after correction:
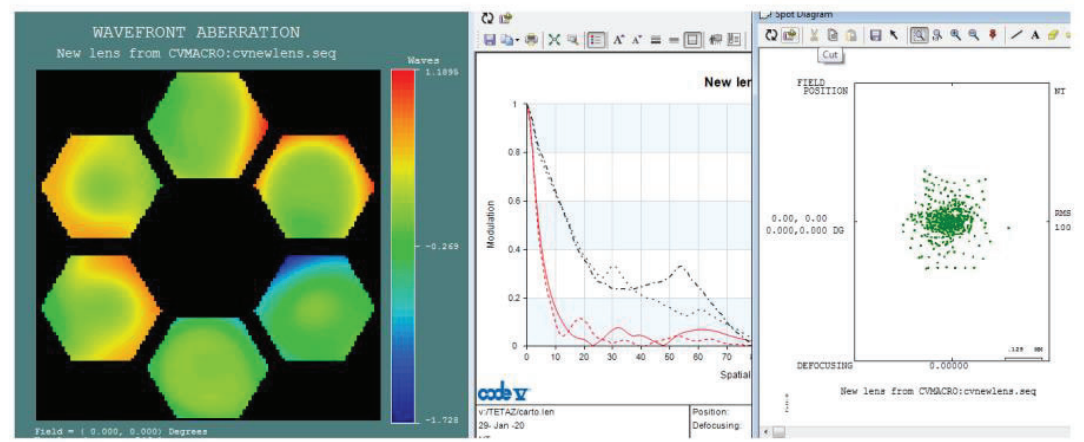

Figure 4: Simulation with CodeV(WFE, FTM, spot diagram) before correction by the DM 
Thus, with Matlab we have simulated the $\mathrm{WFE}_{\text {overall }}$ obtained, after correction with the deformable mirror, for different numbers of actuators on a perfect DM. The purpose of these simulations is to estimate approximately the minimum number of actuators necessary to correct the $\mathrm{WFE}_{\text {overall }}$.

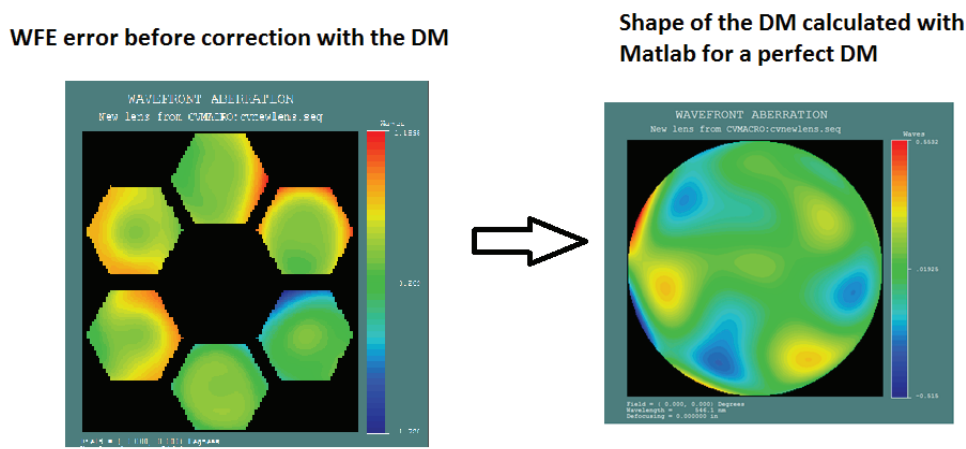

Figure 5: Matlab simulates the best shape of the DM to correct the $W_{F E}$ overall (for different numbers of NLA actuators)

- Comparison between the 3 optical solutions

Hereafter a table which resume the versatility of each optical architecture studied above. Each column represents the maximum authorized value of the parameter considered to hold the final specification MTF shall be $>5$ points at Nyquist. This translates the margin to relax the parameter considered for each optical architecture.

\begin{tabular}{|c|c|c|c|}
\hline & $\begin{array}{c}\text { Maximum } \\
\text { defocus }\end{array}$ & $\begin{array}{c}\text { Maximum decentring/piston/tilt } \\
\text { on M1 petal }\end{array}$ & $\begin{array}{l}\text { Maximum WFE on each } \\
\text { M1 petal }\end{array}$ \\
\hline $\begin{array}{c}\mathrm{N}^{\circ} 3 \text { Segmented DM } \\
\text { (hexapod + NLA) in exit pupil }\end{array}$ & $0.15 \mathrm{~mm}$ & $2 \mu \mathrm{m}$ & 200nm RMS \\
\hline $\begin{array}{c}\mathrm{N}^{\circ} 8 \text { _ Segmented DM (hexapod) in intermediate } \\
\text { pupil + Segmented DM (NLA) in exit pupil }\end{array}$ & $0.15 \mathrm{~mm}$ & $4 \mu \mathrm{m}$ & 100nm RMS \\
\hline $\begin{array}{c}\text { Segmented M2 (hexapod) + Segmented DM in } \\
\text { Exit Pupil (NLA) }\end{array}$ & $0.05 \mathrm{~mm}$ & $1 \mu \mathrm{m}$ & 100nm RMS \\
\hline
\end{tabular}

In conclusion we can say that:

- $\quad\left(N^{\circ} 3\right)$ Segmented DM in exit pupil enables to correct petals polishing error.

- $\quad\left(\mathrm{N}^{\circ} 8\right)$ Solution with 2 accessible pupils enables to correct petals decentering and tilt errors more easily than polishing error.

- Segmented M2 is the worst design, its correction capabilities are too insufficient.

- Mechanical analyses

○ Model description

Below is presented the first study of the FEM under NASTRAN of the telescope GeoHR, that is to say:

- The front cavity is designed with a Si3N4 structure,

- The back cavity is designed with a CFRP envelop

- Excepted for the primary mirror, all the optical parts are represented by punctual masses. The devices supporting the mirrors are simplified thanks to rigid elements.

- All the links in the telescope are modeled by rigid elements. 

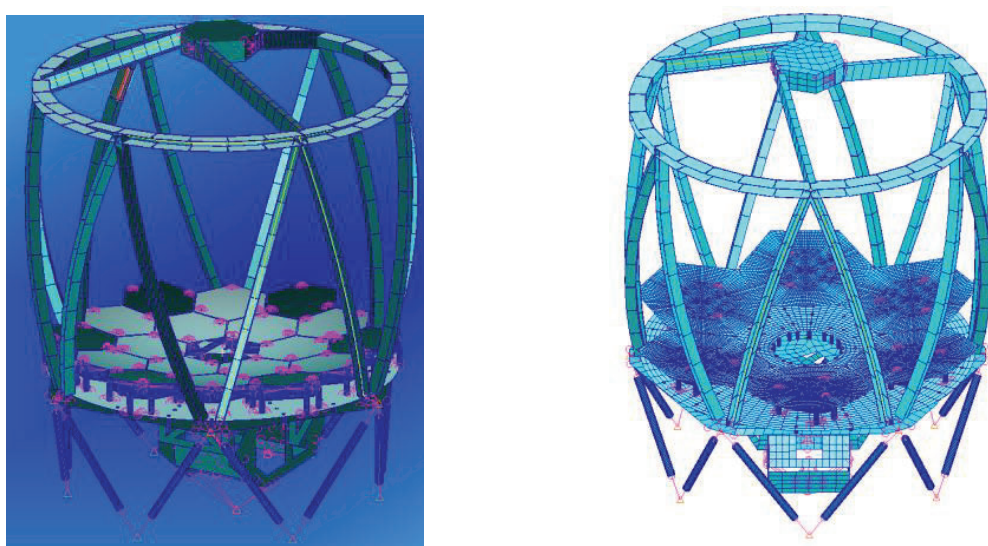

Figure 6: Mechanical models - front cavity in Si3N4 - Left,18 petals; Right, 6 petals telescope

The size of the individual Si3N4 structures, MFD and MLA under the petal are the same than on the 18 petals model.

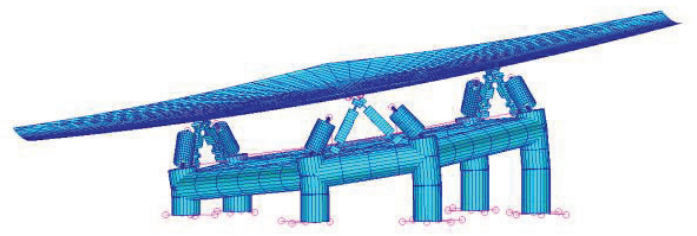

Figure 7: Details of the primary mirror interfaces with Si3N4 structure

- Thermo-elastic analysis

Initial temperature at $20^{\circ} \mathrm{C}$, on nodes of the whole $\mathrm{FE}$ model of the telescope.

\section{Displacements}

Displacement calculated:

\begin{tabular}{|l|l|}
\hline & $Z(\mu \mathrm{m})$ \\
\hline M1_18 petals & 0,22 \\
\hline M1_6 petals & 0,20 \\
\hline
\end{tabular}

\begin{tabular}{|c|c|c|}
\hline$\Delta \mathrm{T}=+1^{\circ} \mathrm{C}$ & $\mathrm{X}(\mu \mathrm{m})$ & $\mathrm{Y}(\mu \mathrm{m})$ \\
\hline $\mathrm{M} 1 \_18$ petals & 0,67 & $\mathbf{0 , 7 2}$ \\
\hline M1_6 petals & $\mathbf{0 , 2 0}$ & 0,15 \\
\hline
\end{tabular}

Table 1: Left Maximum residual gap between 2 petals; Right Maximum displacement in the plane for 1 petal

\section{○ WFE}

The petals nodes are defined in local frames whose $\mathrm{X}$ and $\mathrm{Y}$ axis projected on the interface plane of the telescope are collinear to each other. The WFE is calculated for each of these frames in the largest inscribed circle in the hexagon.
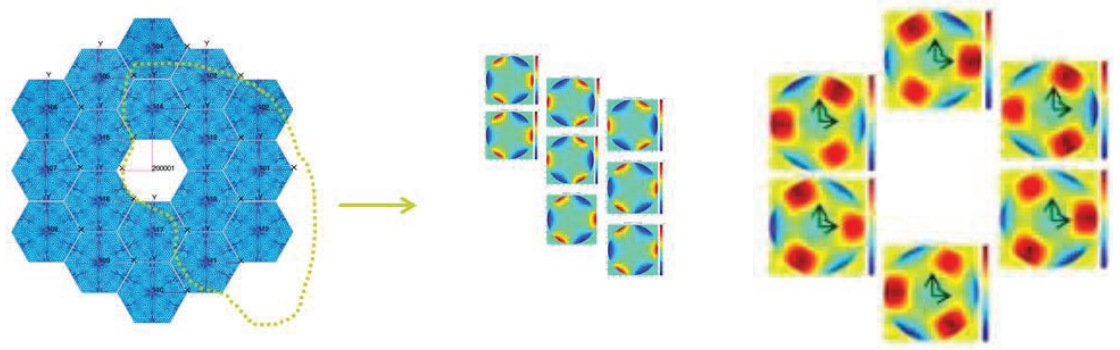

Figure 8: Left WFE M1 18 petals 1,7 nm RMS PTF; Right WFE M1 6 petals 2nm RMS PTF 
ICSO 2020

Thanks to Zerodur ${ }^{\circledR}$ used for the segmented primary mirrors, the thermal impact on the WFE is insignificant.

\subsection{Trade-offs}

- $\quad$ Trade-Off Deformable Mirror

- Existing technologies have been studied and challenged:

- Stacked array mirrors

- Bimorph mirrors

- Unimorph mirrors

- Emecromagnetic voice coil, magnetic mirrors

- MOEMS

- Mechanical actuators

- MADRAS

- FAMRE

- Active carbon shell mirror

- Actuated hybrid mirror

- Trade-Off Wave Front Sensor:

- Shack-Hartmann

- Zernike sensor for extremely low level differential aberrations

- Pyramid wave-front sensot

- Phase diversity

- Pair-wise estimation

○ COFFEE

- $\quad$ Trade-Off M2 Mechanism

○ Articulated Arm

- Telescopic Boom

- Tape Spring on Center

- Tape Spring on periphery

- Truss Mast

- $\quad$ Trade-Off M1 Actuators

○ Piezolectric actuators

- Electromechanical actuators

\subsection{Design Description}

The configuration chosen by the TAS team is that (named $N^{\circ} 8$ ) with a segmented hexapod(MLA) on the first pupil and a segmented DM(NLA) on the second pupil and with a deployable front cavity. Main advantages:

- Efficient to correct petal positions of the primary mirror,

- To separate the function "deformable mirror" and the function "positioning by hexapod" (less industrial and technology risks) for active optics,

- A deployable cavity for the next generation of big size instrument.

The architecture for a GeoHR satellite has been retained to be compliant with the main requirement asking a MTF $>5$ pts at Nyquist frequency. 


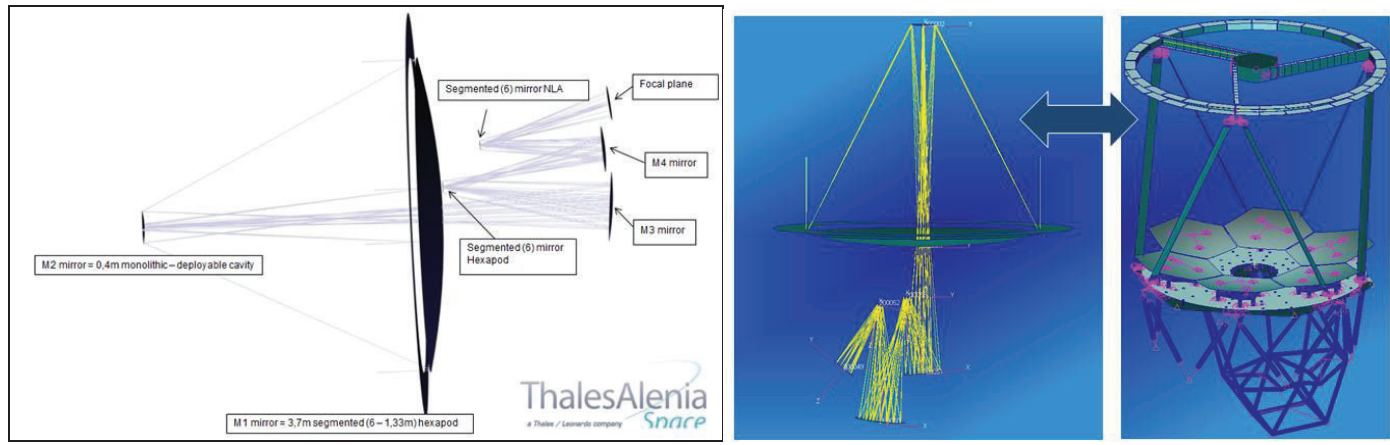

Figure 9: The Optical Architecture retained

This architecture is summarized hereafter it consists in:

- A six-segmented hexagonal primary mirror. Each segment presents a micrometric movement to permit a fine positioning,

- A secondary monolithic mirror,

- A deployable front cavity which permits to roll up itself for launching but also permits in space, once unrolled, to adjust the fine positioning of the secondary mirror,

- A correction of the entry pupil in two pupil planes: intermediate pupil and exit pupil. In both cases the correction is done by segmented mirrors. In intermediate pupil the mirror corrects mainly the line of sight and in the exit pupil the mirror corrects the nanometric defaults after be analyzed by a in-flight phase retrieval algorithm processing,

- Widely using hyper stable materials (carbon, ceramic),

- Using mirrors in Zerodur ${ }^{\circledR}$.

\section{ACTIVE OPTICS IN DEPLOYABLE SYSTEMS FOR SCIENCE}

\subsection{The Astrophysical Context}

To be exhaustive, the science objectives of a Space Observatory[1] with diameter of collecting area $>12 \mathrm{~m}$ are:

Exploring the full diversity of exoplanets / Discovering and characterizing exoplanets in the habitable zones of Sun-like stars and searching for biosignatures in their atmospheres / Remote sensing of the planets, moons, and minor bodies of the solar system / Exploring the building blocks of galaxies both in the local universe and their emergence in the distant past, and elucidating the nature of dark matter / Understanding how galaxies form and evolve from active to passive, both by studying their stars and their gaseous fuel across all temperatures and phases / Following the history of stars in the local volume out to tens of megaparsecs to understand how they form and how they depend on their environment / Observing the birth of planets and understanding how the diversity of planetary systems arises.

The most demanding one is the search and characterisation of exoplanets by direct imaging to allows their atmosphere characterisation [1].

Since about the early 80ths active optics techniques have became essential in large Earth-based telescopes (E-ELT, VLT, GMT, etc.) mainly for Atmophere correction (eXtrme Adaptive Optics). In addition coronagraphy technics allowed suppressing the stellar light. Static residual supperssion and Post-precessing are finishing isolating the flux from the exoplanet:
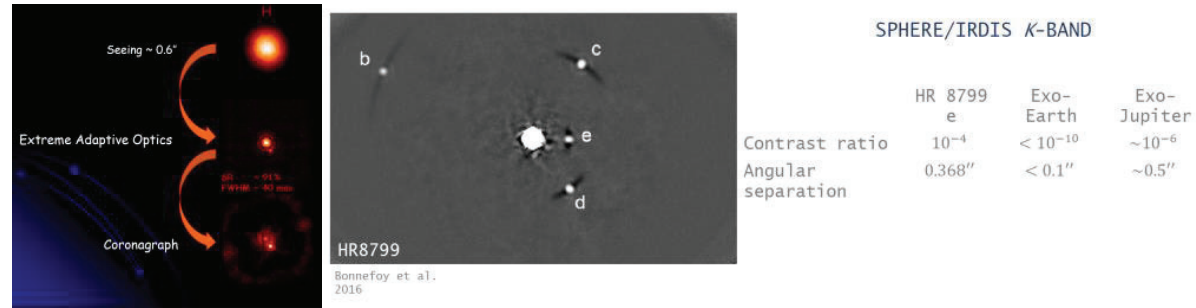

Figure 10: Exemple of results after Extreme adaptative Optics and Coronagraph with SPHERE on VLT. 
If the contrast limitation is around $10^{-6}$ on ground the expectation in Space is about $10^{-10}$ using Apodized Pupil Lyot Coronagraphs (APLC) type with two internal deformable mirrors (DMs) that correct static optical field error to create a "dark hole" and can also correct some wavefront error drift on the order of $0,002 \mathrm{~Hz}$ (10 minute update rate) depending on the target star brightness:

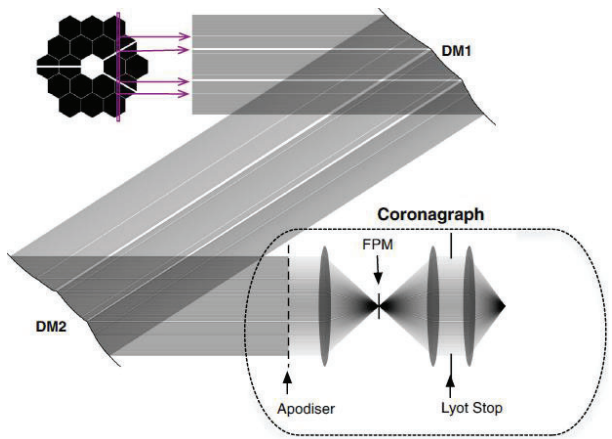

Figure 11: APLC type coronagraph

- Apodizer: It optimizes the distribution of the light beam emitted by the star so that it is blocked by the Focal Plan Mask (FPM) as much as possible.

- FPM: It obscures the light rays emitted by the star

- Lyot Stop: In pupil plane it blocks the diffractive effects due to the shape of the FPM

- Dark Hole : High contrast zone, delimited by the inner and the outter working angle of the high contrast instrument

A coronagraph dark hole is a region of the detector with low background signal that can support high contrast imaging. The dark hole for an APLC is typically annular and centered on the host star under observation, as in Figure 12. The inner radius of the dark hole is called the "inner working angle" and sets the limit for how close a planet can be to its host star and still be detected. The outer radius of the dark hole is called the "outer working angle" and is limited by the spatial resolution of the DMs (approximately the number of actuators across the telescope pupil divided by 2). The phrase "digging the dark hole" refers to the process by which the deformable mirrors are adjusted to correct for static errors in the telescope to create the annular region. This report is focused on stability - or "maintaining the dark hole"
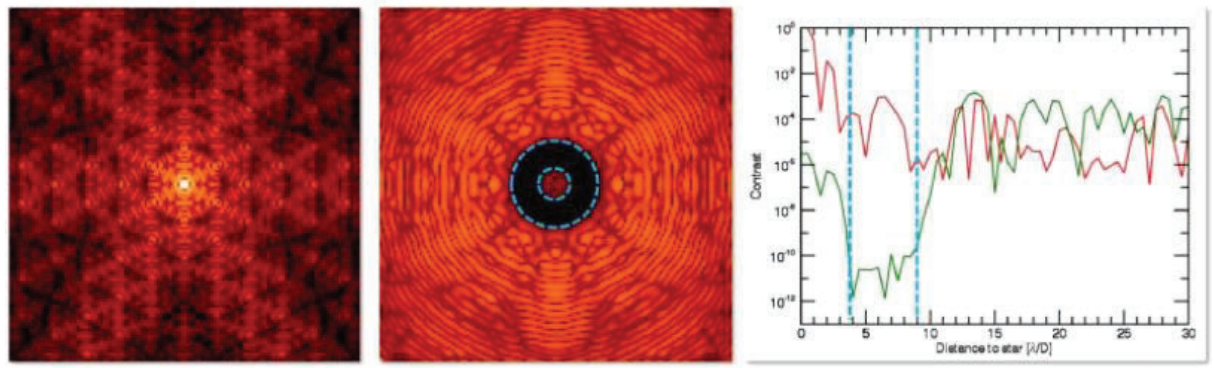

Figure 12: Left: The simulated PSF of a bright star with a segmented telescope and no coronagraph. Center: The coronagraph creates an annular region called the "dark hole" where exoplanets can be detected. Right: PSFs reveal the ability of the coronagraph to create very low contrast $\left(\sim 10^{-11}\right)$ in the dark hole From[2].

In the last 5 years at least scientists have set a few important points:

- The 10-10 high contrast level needed to exoplanet search and characterisation is in theory achievable in space including central, spider and segment gap obscuration and diffraction[5][6][7][8]:

\begin{tabular}{|l|l|l|l|}
\hline Obscuration Specifications & \% of the M1 diameter & Calculation for a M1 of 16m & $\begin{array}{l}\text { TAS Design proposal with a } \\
\text { M1 of } 16,7 \mathrm{~m}\end{array}$ \\
\hline Central Obscuration & Diameter $<15 \%$ & $\mathbf{2 , 4 m}$ & $\mathbf{2 , 1 4 m}$ \\
\hline Spiders Obscuration & Thickness $<1 \%$ & $\mathbf{1 6 c m}$ & $\mathbf{1 2 c m}$ \\
\hline Segment Gaps & Dimension $<0.1 \%$ & $\mathbf{1 , 6 c m}$ & $\mathbf{1 c m}$ \\
\hline
\end{tabular}


- The issue will be the stability knowing that we will be able to calculate tolerances of positionning for spatial frequencies of each Primary Mirror segment and by inversion directly set the spatial correction to apply for each segment thanks to tools like PASTIS [11] and Spatial/Temporal impact taken from ULTRA analysis[13]
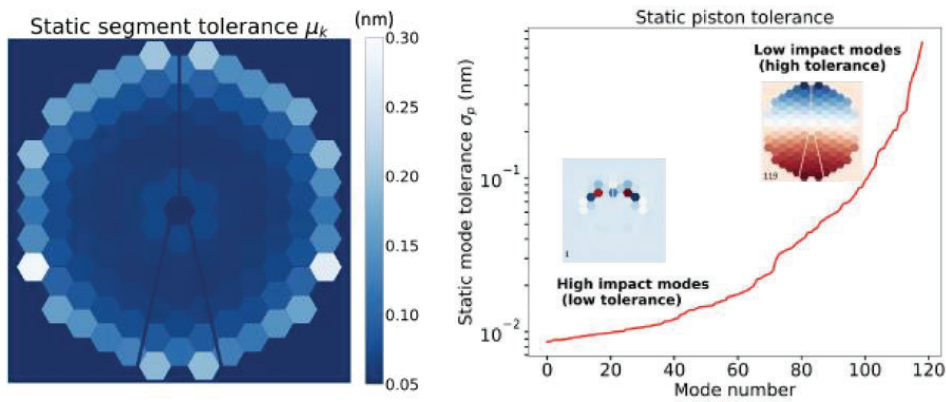

Figure 13: Static per-segment piston tolerances for the LUVOIR-A $15 \mathrm{~m}$ telescope with the baseline coronagraph design for local piston aberrations and a target contrast of ctarget $=10^{-10} \cdot[11]$

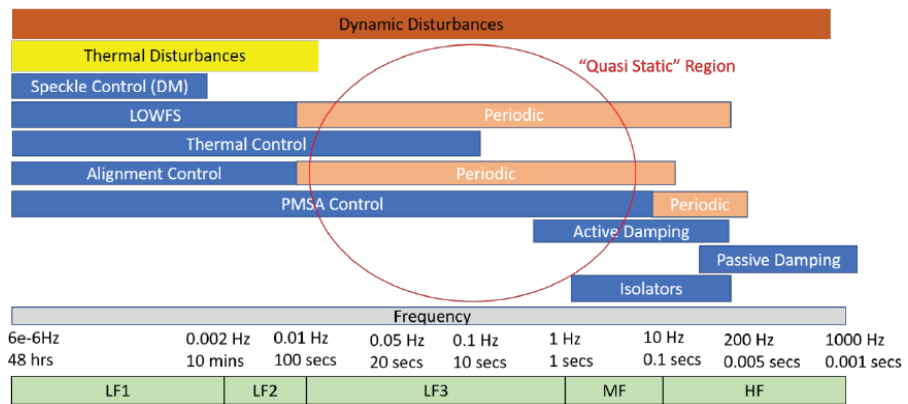

Figure 14: Expected disturbances and notional control systems in the temporal domain. The green bar represents the notional bandwidths of the frequency "bins". The "quasi static" region is of highest concern since the coronagraph is most sensitive to errors in this frequency regime and there are fewer active/passive controls [13]

- To set the time tolerance of stability drift and directly set the time needed between correction to not impact the observation Thanks to tools like the macro developped by the ONERA (Excel file delivered in the frame of the MOD4) from ULTRA analysis giving the acceptable drift by modes from PASTIS calculation of modes sensitivity [11][13]:

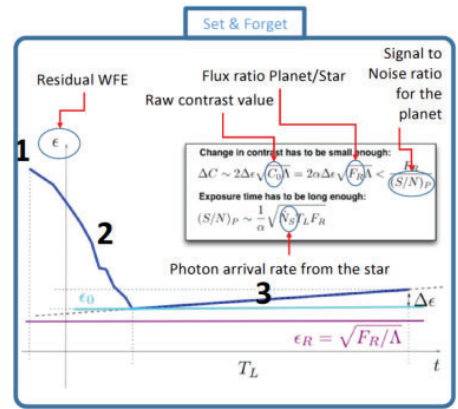

\begin{tabular}{|l|l|c|l|}
\hline \multicolumn{1}{|c|}{ INPUTS } & \multicolumn{2}{l|}{} \\
\hline Exposure time & seconds & 3600 & Scientific exposure time required to see a planet \\
\hline Flux ratio & & $1,00 \mathrm{E}+10$ & Ratio of peak flux between star and planet \\
\hline SNR & & 10 & Signal to noise desired for the planet \\
\hline C0 & & $2,00 E-11$ & Floor contrast \\
\hline WFE & pm & 100 & Aberration RMS value (epsilon 0 in the note) \\
\hline Star flux & Nphoton/s & $1,00 E+10$ & Photon flux of the star, on the entire telescope pupil \\
\hline \multicolumn{1}{|c|}{ OUTPUTS } & & & \\
\hline Contrast & & $1,39 E-09$ & Contrast value reached with WFE and C0 \\
\hline FR & & $1,00 E-10$ & Flux ratio of the planet \\
\hline EpsilonR & pm & 27 & Equivalent WFE for planet signal \\
\hline Alpha & & 3,7 & Ratio WFE / EpsilonR \\
\hline Drift & $\mathrm{pm} / \mathrm{s}$ & 0,008 & Maximum acceptable drift of WFE \\
\cline { 2 - 5 } & $\mathrm{pm} / \mathrm{h}$ & 29 & Maximum acceptable drift of WFE \\
\cline { 2 - 5 } & $\mathrm{pm} / 10 \mathrm{~h}$ & 294 & Maximum acceptable drift of WFE \\
\hline
\end{tabular}

Figure 15: Time evolution of wavefront error for "Set and Forget" on the left and ULTRA appication on this drift calculation on the right. 


\subsection{Proposed Science case architecture and solutions}

A space observatory LUVOIR type for such mission as exoplanet charactisation shall be focused on:

- Maximisation of the primary mirror collecting area which will maximise SNR

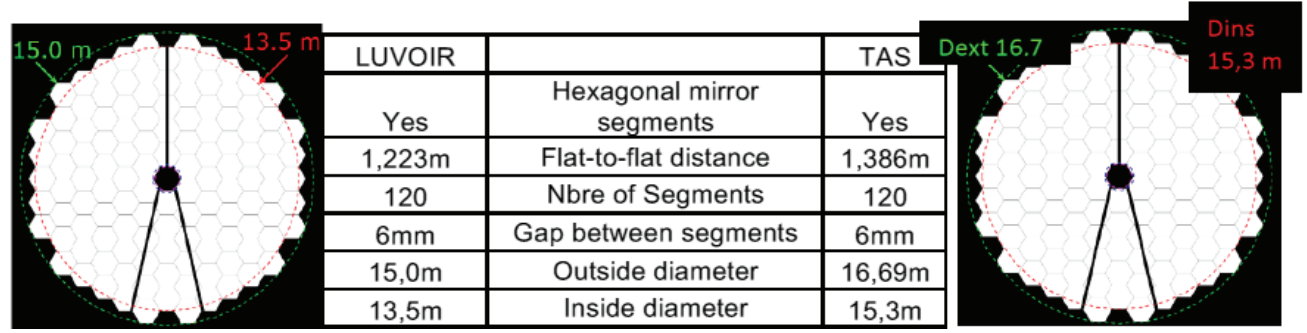

- Maximisation of the Primary Mirror (PM) performance (more than $80 \%$ of the observatory performance is on the PM) by cleaning as much as possible the WFE at segment level to guaranty the global WFE of the instrument $<38 \mathrm{~nm}$ RMS.

- Maximize the stability

- Highly simplified integration and calibration On Ground and In Orbit

- No Need of extra Deformable Mirror on the Instrument (only at Coronagraph level) which could avoid having an intermediate pupil in the telescope optical train, thus reducing the number of mirrors.

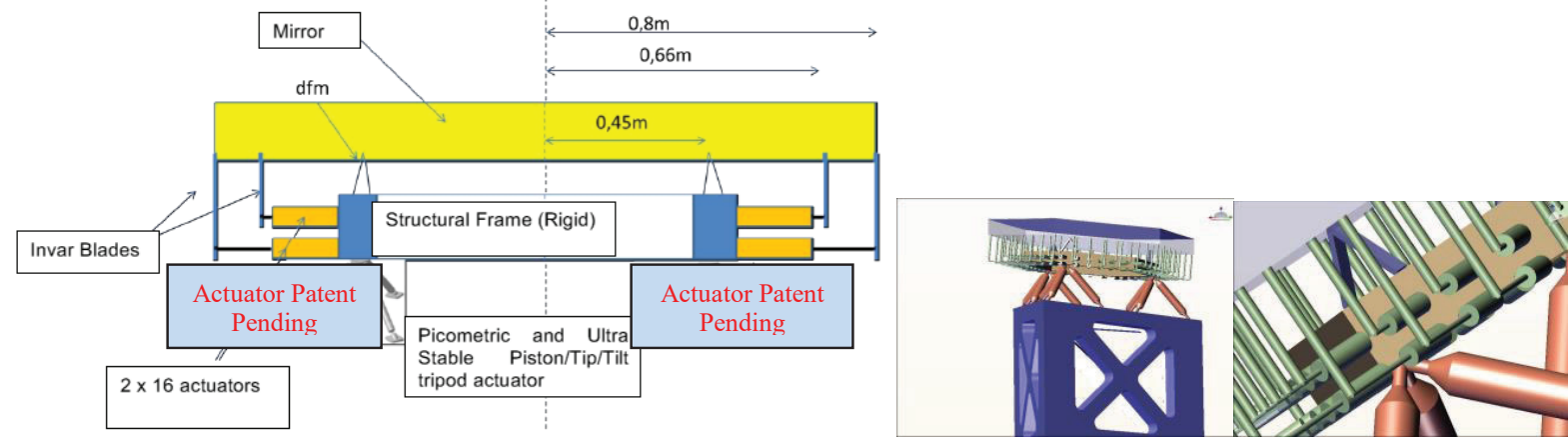

Figure 16: PM Active WFE Segment and Ultra Stable Picometric Piston/Tip/Tilt tripod Actuator

Principle:

- The Mirror is held by 3 DFM, themselves fixed on a structural frame

- Actuators on 2 diameters of the mirror will deform the mirror by pushing on Invar Blades

- Picometric and ultra stable Piston/Tip/Tilt tripod actuators hold the structural frame and are used to move and correct at picometric level with a very high stability the segment mirror group

- A rigide and structural frame make the link between the actuators, the DFM and the picometric actuators

Working directly on a picometer displacement $\&$ high stability is directly facing the physic laws, for reminder atomic radius ofHydrogen $(\mathrm{H} 1)=53 \mathrm{pm}$. The use of a huge Sunshield in L2 will guaranty a very high level of thermal stability $(\sim \mathrm{mK})$, allowing to use the intrinsic thermal material stability. Smart structural design and characterisation will make it fit to requirements by adjusting temperature compatible with acceptable drift and time correction. Advantages:

- Naturally stable to be as close as possible in the Set\&Forget case

- Drift correction compatible with the coronagraph tolerance and imaging process capabilities or Machine Learning with AI.

- Characterisation of the overall Satellite and Observatory dynamics to anticipate the impact of the constant pointing and applying corrections with no impact on the Dark Hole contrast digging. 
Primary Mirror Deployment: A Two Parts Option

Looking forward simplification and efficiency the PM shall be Symetrical to ease optical and postprocessing correction, no-gap articulations for simplified on-ground end-stop calibration and in-orbit motorization control.

\begin{tabular}{|c|c|c|c|c|c|c|c|}
\hline \multirow[t]{2}{*}{ Launcher / Design TAS } & \multicolumn{3}{|l|}{ Fairing } & \multicolumn{2}{|c|}{ TAS Design 2 parts } & \multicolumn{2}{|c|}{ TAS Design 3 parts } \\
\hline & Diameter & Height & Capacity to GTO & $\begin{array}{l}\text { Overall Size in } \\
\text { fairing }\end{array}$ & \begin{tabular}{|l} 
M1 \\
Diameter
\end{tabular} & $\begin{array}{l}\text { Overall size } \\
\text { in fairing }\end{array}$ & $\begin{array}{l}\text { M1 } \\
\text { Diameter }\end{array}$ \\
\hline Ariane 64 & $5,4 \mathrm{~m}$ & $20 \mathrm{~m}$ & 12 tons & $4,9 \mathrm{~m}$ & $9,0 \mathrm{~m}$ & $5,3 \mathrm{~m}$ & $7,2 \mathrm{~m}$ \\
\hline Blue Origine New Glenn & $7,0 \mathrm{~m}$ & & 13 tons & $6,3 \mathrm{~m}$ & $11,6 \mathrm{~m}$ & $6,8 \mathrm{~m}$ & $9,3 \mathrm{~m}$ \\
\hline SLS Block2 & $7,5 \mathrm{~m}$ & $24,8 \mathrm{~m}$ & 45 tons & $6,8 \mathrm{~m}$ & $12,5 \mathrm{~m}$ & $7,3 \mathrm{~m}$ & $10 \mathrm{~m}$ \\
\hline SpaceX Starship Current & $9 \mathrm{~m}$ & $19 \mathrm{~m}$ & 100 to 150 tons $*$ & $8,1 \mathrm{~m}$ & $15,0 \mathrm{~m}$ & $8,8 \mathrm{~m}$ & $12 \mathrm{~m}$ \\
\hline SpaceX Starship Evolution & $>9 \mathrm{~m}$ & $>19 \mathrm{~m}$ & 100 to 150 tons $*$ & $9,4 \mathrm{~m}$ & $16,7 \mathrm{~m}$ & $12,2 \mathrm{~m}$ & $16,7 \mathrm{~m}$ \\
\hline
\end{tabular}

*refueling in orbit

Table 2: TAS 2 and 3 parts Mirror Design scaled to Launcher options

Hereafter some 3D view of the TAS 2 parts design in stowed and deployed configuration:
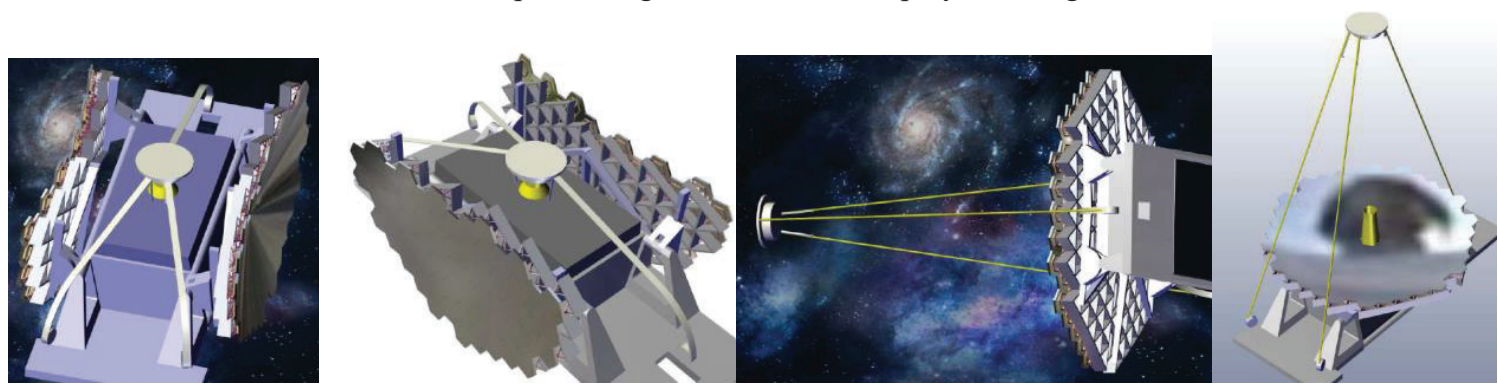

Figure 17: TAS 2 parts Telescope in stowed (left) and deployed (right) configuration

For the size of alf the PM diameter we can fit all the instrument and minize number of deployment and associated risks. The M2 is first deployed with the 3 tape spring used also as Secondary Mirror pointing mechanism. The central baffle is deployed after the M2. Finaly the two parts of the M1 are deployed and locked:

- Deployment of M2

The TAS solution is based on the 3 Tape Springs Deployment technologies with the following advantages:

- $\quad$ Stowed configuration simplified, the M2 will be directly stowed on the central structure

- Smooth and controlled deployment with less risks of blocking and seizure than articulated beams

- Active Piston/Tip/Tilt correction when deployed (Accuracy 100nm/Stroke $100 \mu \mathrm{m} / \mathrm{Stability} 100 \mathrm{~nm}$ RMS/s)

- $\quad$ Supporting Structure:

Future structure will be a compromise of "smart" structure as "auxetic" shape to counterbalance thermoelastic and mechanical deformation during motion of the complete observatory and adjustment capabilities of the active segments (WFE and Piston/Tip/Tilt).

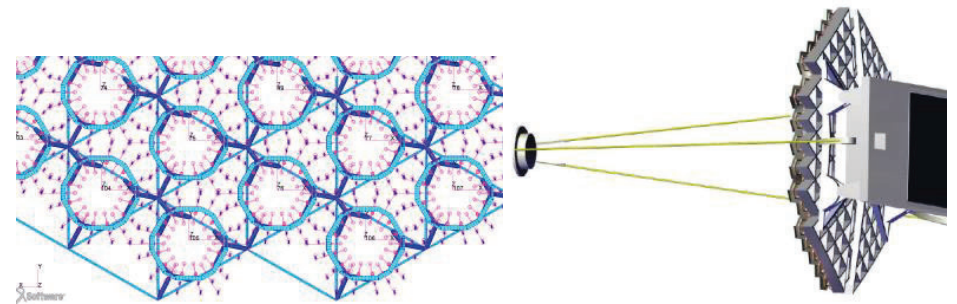

Figure 18: View of the structural frame / Picometric Tripod / Supporting Structure arrangement 
CAD model thermo-mechanical sensitivity and WFE impact first results:

\begin{tabular}{|l|l|l|}
\hline & & \\
\hline
\end{tabular}

Table 3: Thermo-mechanical impact on TAS design options

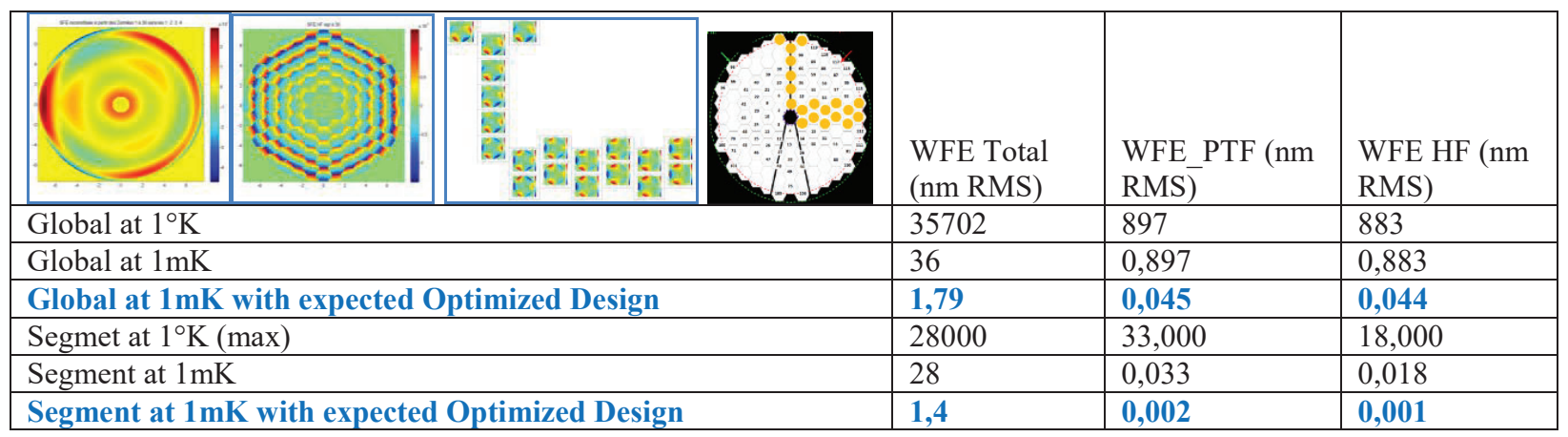

Table 4: WFE calculation and expected for optimized TAS design

No issues on both needed corrections and impact of thermal stability on the primary mirror WFE. 


\section{SYNTHESIS AND CONCLUSIONS}

\subsection{On GEO-HR Case}

Such an instrument will have on board correction of micrometric level mirrors (hexapod allowing an overall correction in piston and tip / tilt) but also at the nanometric level (optimization of the mirror surface).

- Associated Road Maps

There are four technological products essential to the realization in the near future of the first high resolution instrument for monitoring the earth in real time and subjected to two dedicated unit validation:
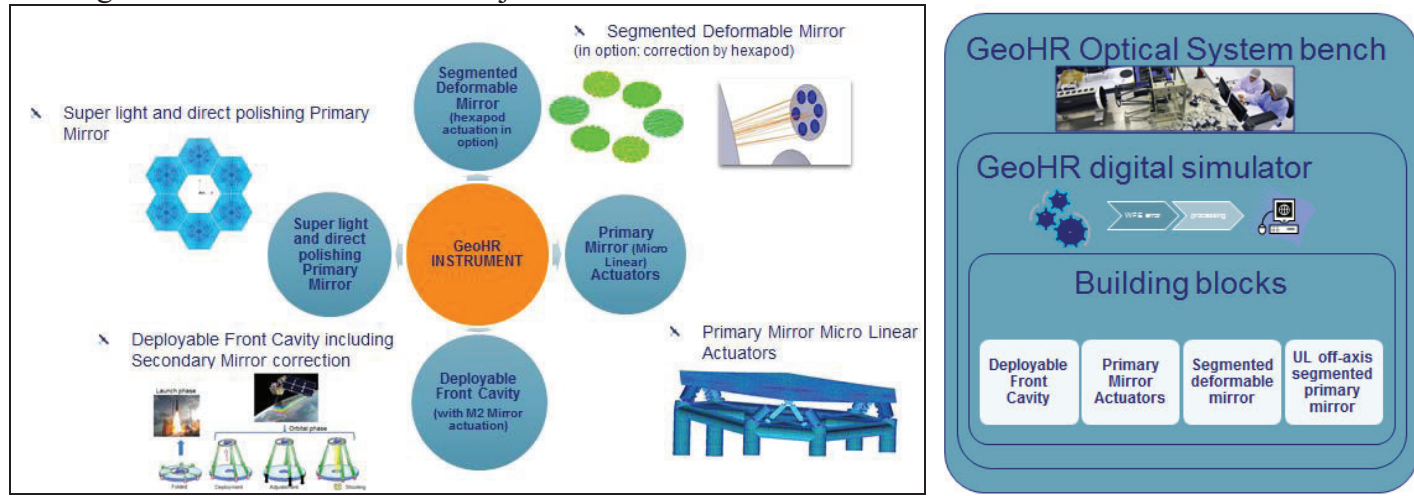

Figure 19: Essentials Building Blocks \& System Validation

Technology Building Blocks

○ Segmented Deformable Mirror

- Deployable Front Cavity

Primary Mirror (micro linear) Actuators

- Super Light and Direct polishing segmented off-axis primary mirror

Units Validation

- Metrology System at actuator level \& at "equipment" level (for instance M1, deformable mirror...)

○ Digital Simulator and Optical System Bench

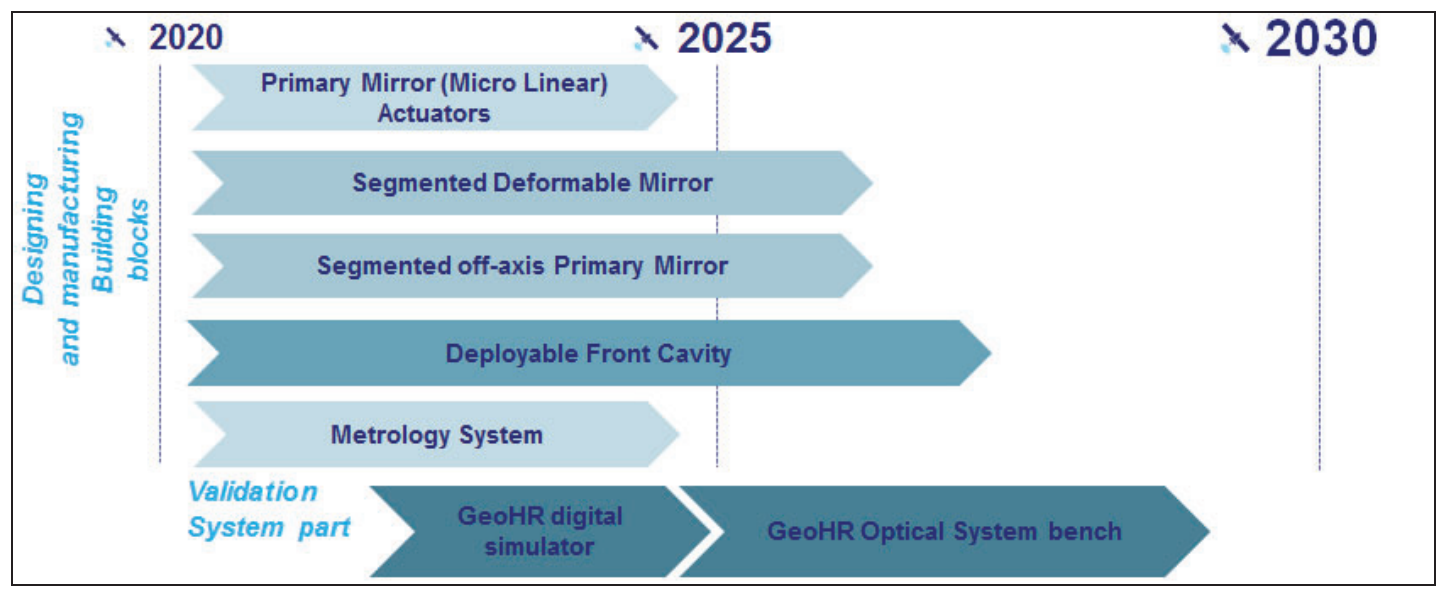

Figure 20: Necessary Developments Roadmap 


\subsection{On Science Case}

Way to proceed to reach the extreme requirements of exo-earth direct characterisation on Space Observatory such as LUVOIR-A:

- From Scientists last outputs needs:

○ Extremely High Contrast level Coronagraph (10-10)

- Extrem Spatial \& Temporal Stability $(10 \mathrm{pm} / 10 \mathrm{mn})$

- A Telescope Architecture proposal for extremely passive stability

- Set\&Forget case: Optical correction on Primary Mirror Segments \& Limit activation and drift using intrinsic stability characterisation

- A Sytem control directly from the Coronagraph processed images

- PASTIS for correction of the Segment Piston/Tip/Tilt

- Aberration drift calculation by ULTRA analysis principle

- Architecture Characterisation

- Image Processing (Image accumulation, de-convolution, AI and Machine learning)

The global approach is simplifed to an intrinsic stability observatory needed for such missions:
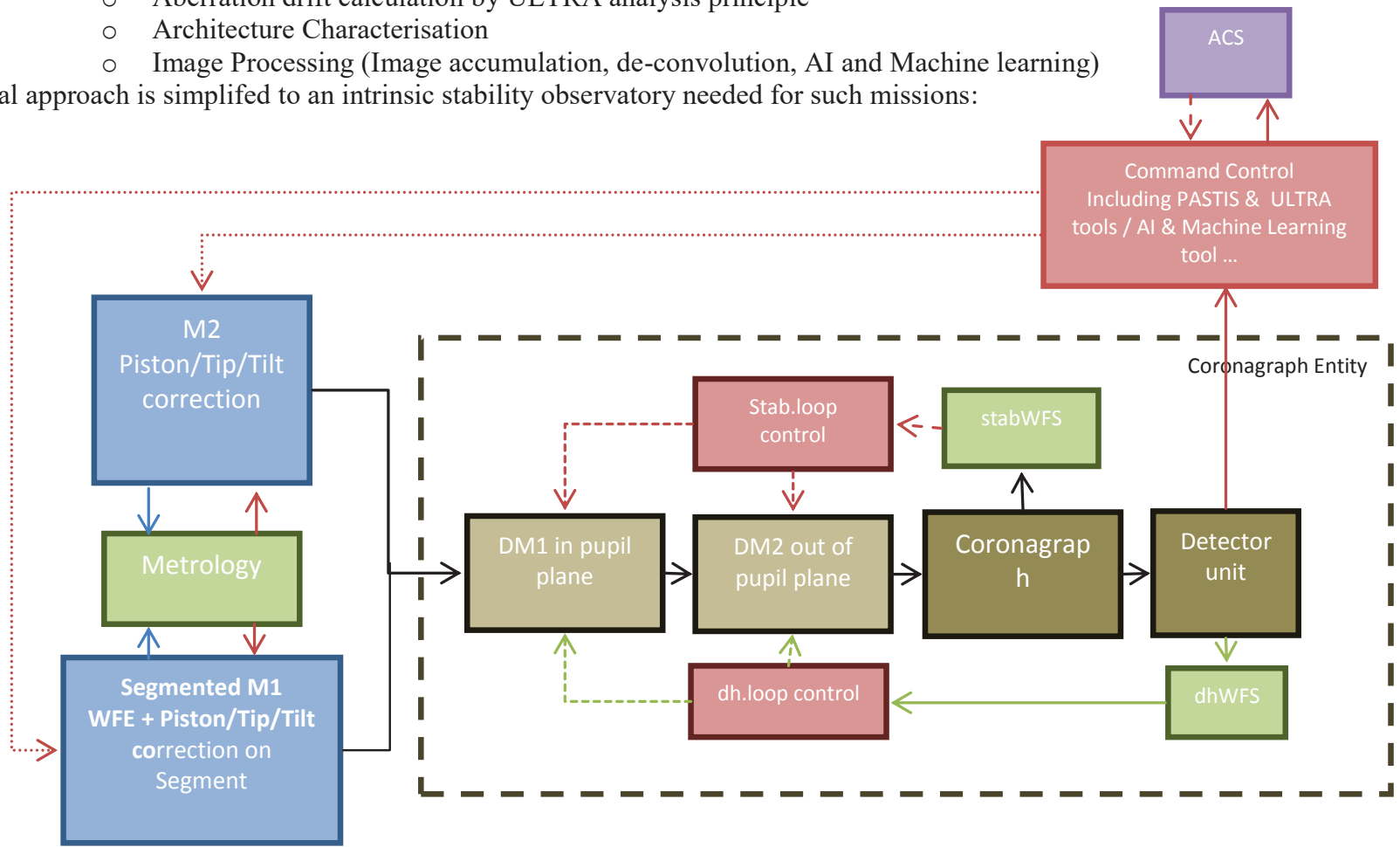

The strategy:

Step 1: Deployment of the M2 then M1, using internal metrology (capacitive, inductive, optical ...) for first positioning (under $100 \mathrm{~nm}$ )

Step 2: Set the Coronagraph: Images \& ACS tools to adjust the M1 \& M2 positionning (pm level)

Step 3: Dig the Dark Hole: Using the images to adjust the M1 (pm thermal adjustment) \&Coronograph internal loop Step 4: Observation time: M1 correction anticipation (set\&forget pm stability) \& Coronograph internal loop control

- $\quad$ Associated Road Maps:

- $\quad$ Upgrade PASTIS model and ULTRA macro to low order Zernike

- $\quad$ Attitude Control System \& FSM removal to developp an approach based on Vibration Isolation and Precision Pointing System (VIPPS) + Extremely fine and fully characterised ACS (Magnetic Bearing Sustented Control Moment Gyroscopes, cold gaz thrusters, ...)

- $\quad$ On Board Image Processing to compensate observatory drifts (including Machine Learning \& AI)

- $\quad$ M2 \& Central Baffle Deployment to finalise Tape Spring Design and performances 
- $\quad$ Active M1 Segment Developpments with Ultralight Mirror Shape definition and WFE active correction segment finalisation

- $\quad$ Finalise Picometer Tripod Actuator

- $\quad$ Supporting Structure Shape and Material

The associated schedule for development and validation of the new technologies:

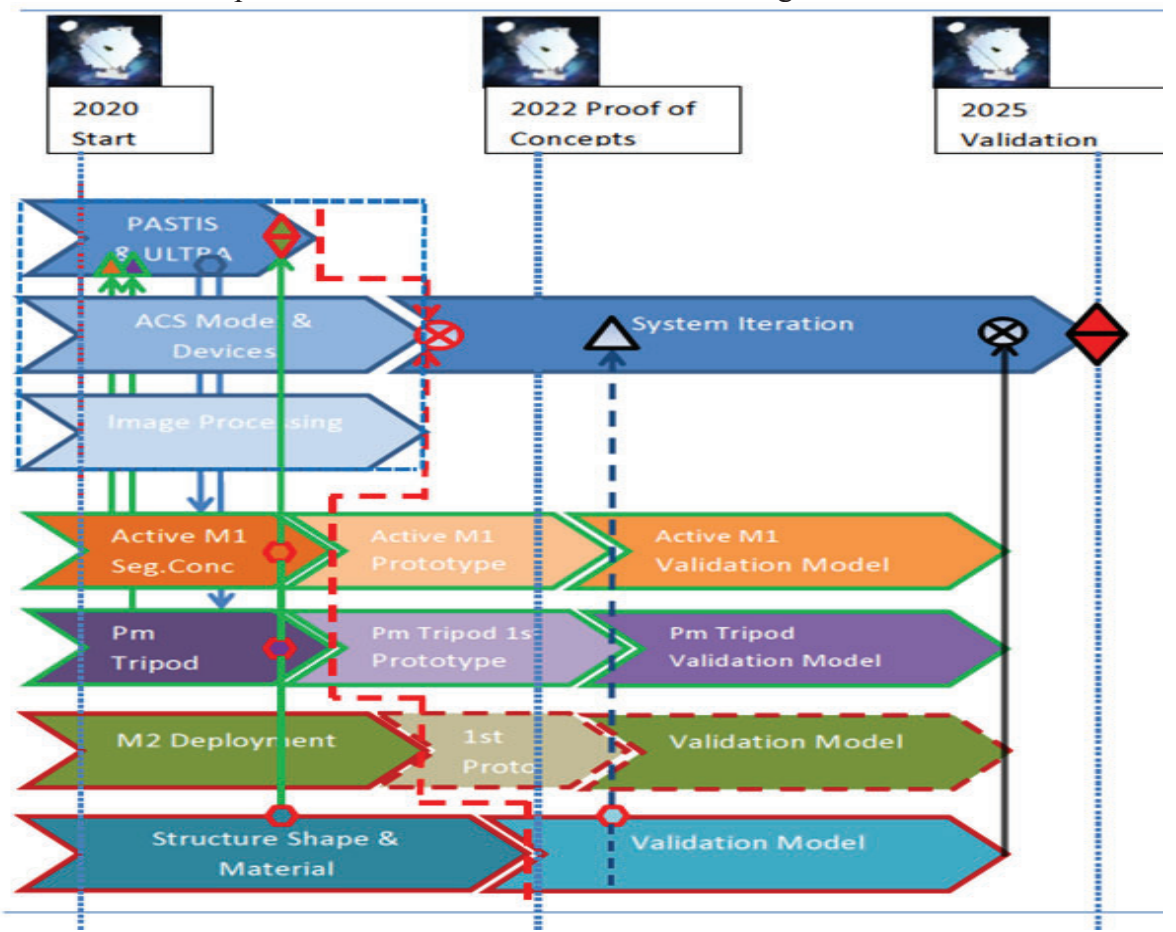

\section{Acknowledgments}

The authors gratefully acknowledge support for the work provided by the European Space Agency under contract number 4000125154/18/NL/AR/ig "ACTIVE OPTICS IN DEPLOYABLE SYSTEMS FOR FUTURE EO AND SCIENCE MISSIONS". 


\section{REFERENCES}

[1] The LUVOIR Final Report 2019-08-26_NASA.

[2] Coyle 2019 : Large ultra-stable telescope system study.

[3] Pueyo \& Norman ApJ 2013: https://iopscience.iop.org/article/10.1088/0004-637X/769/2/102

[4] Guyon et al. ApJ 2014: https://iopscience.iop.org/article/10.1088/0004-637X/780/2/171

[5] N'Diaye et al. ApJ 2016: https://iopscience.iop.org/article/10.3847/0004-637X/818/2/163

[6] Ruane et al. JATIS 2018: https://ui.adsabs.harvard.edu/link gateway/2018JATIS...4a5004R/doi:10.1117/1.JATIS.4.1.015004

[7] ZImmerman et al. SPIE 2016: https://ui.adsabs.harvard.edu/link gateway/2016SPIE.9904E..1YZ/doi:10.1117/12.2233205

[8] Mazoyer et al. 2018a: https://iopscience.iop.org/article/10.3847/1538-3881/aa91cf

[9] Mazoyer et al. 2018b: https://iopscience.iop.org/article/10.3847/1538-3881/aa91d7

[10] Iva Laginja 2019 : Trade-Off analysis and Preliminay Design of the Active Optics System for the Science mision case

[11] Leboulleux, Sauvage, A. Pueyo, etc. : Pair-based Analytical model for Segmented Telescopes Imaging from Space for sensitivity analysis

[12] System Level Segmented Telescope Design (SLSTD) Final Report Rev B_2019-04-24_Lockkeed Martin Advanced Technology Center - Collins Aerospace - Harris Corporation - Coherent

[13] Ultra-Stable Telescope Research and Analysis (ULTRA) Program NASA ROSES 2017 D.15 Award Phase 1 Report_April 1, 2019_L. Coyle, M. East, J. Arenberg, T. Lawton, M. Bluth, L. Puyeo

[14]EOP-SF/2013-09-1757iss1_GEO-HR: Mission Assumption and preliminary Technical Requirements (MATER)

[15] Astronomical Optics and Elasticity Theory - Active Optics Methods; G. Lemaitre, 2008; A\&A library, Springer Ed.

[16] Robert A. Gonsalves, "Phase Retrieval And Diversity In Adaptive Optics," Opt. Eng. 21(5) 215829 (1 October 1982) https://doi.org/10.1117/12.7972989

[17] Projet RASCASSE : Arnaud Liotard, Marc Bernot, Mikaël Carlavan, Frédéric Falzon, Thierry Fusco, Vincent Michau, Aurélie Montmerle-Bonnefois, Laurent Mugnier, Céline Engel, Clément Escolle, Marc Ferrari, Emmanuel Hugot, Thierry Bret-Dibat, David Laubier, "Wave-front sensing for space active optics: Rascasse project," Proc. SPIE 10563, International Conference on Space Optics — ICSO 2014, 105632W (17 November 2017); https://doi.org/10.1117/12.2304111

[18] Optique active sur démonstrateur : Damien Sucher, Guillaume Butel, Guillaume Briche, Jean-François Blanc, Arnaud Liotard, Marc Bernot, Mikaël Carlavan, Aurélien Suau, Nisrine Louh, Lauriane Galtier, Sebastien Guionie, Thierry Viard, Stéphanie Behar-Lafenetre, Fabrice Champandard, Jean-Bernard Ghibaudo, Vincent Costes, "Active optics for space telescopes," Proc. SPIE 11116, Astronomical Optics: Design, Manufacture, and Test of Space and Ground Systems II, 1111611 (9 September 2019); https://doi.org/10.1117/12.2529103 